\title{
On the Performance of Quantum Key Distribution FSO Systems Under a Generalized Pointing Error Model
}

\author{
Hui Zhao, Student Member, IEEE, and Mohamed-Slim Alouini, Fellow, IEEE
}

\begin{abstract}
In this letter, the performance of a quantum key distribution (QKD) free-space optical (FSO) system is analyzed while taking a generalized pointing error model into account. More specifically, closed-form expressions for the average received powers at both the legitimate receiver and eavesdropper are derived. In addition, their corresponding asymptotic results valid in the high telescope gain regime are also presented. To capture the secure performance, we also investigate the ratio of received powers at the eavesdropper and at the legitimate receiver. Further, in some special cases, we find the optimal telescope gains for the received powers at both the legitimate receiver and eavesdropper, as well as the power ratio, which is important and useful for a secure QKD FSO system design. Finally, some selected numerical results are presented to illustrate the mathematical formalism and validate the accuracy of the derived analytical expressions.
\end{abstract}

Index Terms-Free-space optical, generalized pointing errors, secure performance, and quantum key distribution.

\section{INTRODUCTION}

To achieve confidential communications, a one-time-pad scheme was proposed by [1], where a secret key is shared between two legitimate parties. Due to the reliance only on the computational complexity, this traditional key distribution method is fundamentally insecure [2]. This is particularly true with the fast development of large-scale quantum computers over the last decade, which is making the current public key infrastructure more and more vulnerable [3]. In this context, quantum key distribution (QKD), a promising application of cryptography, provides unconditional security based on the law of quantum physics and quantum non-cloning theorem [4]. QKD can be implemented in two kinds of medium, namely optical fiber and free-space optical (FSO) links. Due to its cost-effectiveness, high-bandwidth availability, deployment flexibility and interference-immunity, FSO is an excellent choice for the terrestrial backhaul solution [1] and the feeder link of very high throughput satellites [2]. However, in the FSO systems, stochastic jitter and vibration of the pointing

Manuscript Received June 15, 2019; accepted July 9, 2019. This work was funded by the office of sponsored research (OSR) at KAUST. The work of $\mathrm{H}$. Zhao was done while he was studying at KAUST. The associate editor coordinating the review of this paper and approving it for publication was M. Koca. (Corresponding author: Hui Zhao.)

H. Zhao was with the Computer, Electrical, and Mathematical Science and Engineering Division, King Abdullah University of Science and Technology (KAUST), Thuwal 23955-6900, Saudi Arabia, and he is now with the Communication Systems Department, EURECOM, Sophia Antipolis 06410, France (email: hui.zhao@kaust.edu.sa).

M.-S. Alouini is with the Computer, Electrical, and Mathematical Science and Engineering Division, King Abdullah University of Science and Technology, Thuwal 23955-6900, Saudi Arabia (email: slim.alouini@kaust.edu.sa).

Color versions of one or more of the figures in this paper are available online at http://ieeexplore.ieee.org.

Digital Object Identifier direction caused by atmospheric turbulence or building sway should be taken into account [5], [6], [7].

An inevitable drawback in the QKD FSO systems is backflash in the legitimate receiver side, when the legitimate receiver adopts a common detection method, relying on a single photon avalanche photodiode (SPAD) [8], [9], [10]. Indeed, with the SPAD detection scheme, an avalanche happens, resulting in the emission of a secondary photon, i.e., backflash, which can be captured by eavesdroppers. Kupferman and Arnon in [10] have recently presented an analytical framework to evaluate the impact of backflash on the performance of QKD FSO systems. However, in their investigation, the adopted model for the azimuth and elevation pointing error angles assumed zero mean and equal variance for both angles, and the pointing error angle at eavesdroppers was set to zero. However, for real life deployment, QKD FSO systems may have to operate under different and more general conditions. Further, the authors in [10] did not investigate the optimal point of the telescope gain to maximize the received power at the legitimate receiver.

In this letter, we consider a generalized pointing error model, in which the azimuth and elevation pointing error angles at the legitimate receiver have arbitrary and different mean and variance. In addition, we do not limit the pointing error angle at the eavesdropper to be very close to zero. Under this general setup, we are still able to offer closed-form expressions for the average received powers at both the legitimate receiver and eavesdropper. Moreover, the corresponding maximum point of the telescope gain are found in some special cases. To simplify the expressions and get obviously some additional insights, we also derive simple asymptotic expressions when the telescope gain is sufficiently large. Finally, the ratio of the received powers at the eavesdropper and legitimate receiver is analyzed, and the corresponding extreme point of the telescope gain is also obtained in a particular case of interest.

\section{SySTEM MODEL}

In an QKD FSO system, an absolutely static source $(S)$ transmits an information photon to a destination $(D)$ on a vibrating platform (such as a laser satellite), resulting in a random pointing error at $D$. Due to the avalanche of the SPAD detection method, a secondary photon will be emitted, called backflash, which can be detected by a third party, i.e., eavesdropper $(E)$. This communications scenario is presented in Fig. 1 of [10].

The pointing error angle $(\theta)$ at $D$ can be divided into two parts, i.e., $\theta=\sqrt{\theta_{V}^{2}+\theta_{H}^{2}}$, where $\theta_{H}$ and $\theta_{V}$ are the azimuth and elevation pointing error angles, respectively. Like in [10], we assume that $\theta_{H}$ and $\theta_{V}$ are Gaussian random variables (RVs). However, we consider the general case in which these 
RVs are not necessarily with zero mean and the same variance. That is $\theta_{V} \sim \mathcal{N}\left(\mu_{V}, \sigma_{V}^{2}\right)$ and $\theta_{H} \sim \mathcal{N}\left(\mu_{H}, \sigma_{H}^{2}\right)$, where $\mu_{V}$ and $\sigma_{V}^{2}$ (or $\mu_{H}$ and $\sigma_{H}^{2}$ ) are the mean and variance of $\theta_{V}$ (or $\theta_{H}$ ). In this case, it is well known that $\theta$ follows the Beckmann distribution [11], where the moment-generating function (MGF) of $\theta^{2}$ is known to be given by [11]

$$
\mathcal{M}_{\theta^{2}}(s)=\mathbb{E}\left\{\exp \left(s \theta^{2}\right)\right\}=\frac{\exp \left(\frac{\mu_{V}^{2} s}{1-2 \sigma_{V}^{2} s}+\frac{\mu_{H}^{2} s}{1-2 \sigma_{H}^{2} s}\right)}{\sqrt{\left(1-2 \sigma_{V}^{2} s\right)\left(1-2 \sigma_{H}^{2} s\right)}}
$$

where $\mathbb{E}\{\cdot\}$ represents the average operator. By setting some specific values for the parameters of the MGF of the Beckmann distribution in (6) of [12], the form of (1) can be also easily obtained.

\section{ReCEIVED Power AT $D$}

From [5], [10], the received power at $D$ is given by

$$
P_{D}(\theta)=K_{1} G_{D} L(\theta),
$$

where $G_{D}$ is the telescope gain of $D$ and $L(\theta)=$ $\exp \left(-G_{D} \theta^{2}\right)$ is the corresponding pointing loss factor. In (2), $K_{1}$ is constant, depending on the system characteristic, given by

$$
K_{1}=\eta_{q} P_{S} \eta_{S} \eta_{D} \frac{L_{A}\left(D_{1}\right)}{D_{1}^{2}}\left(\frac{\lambda}{4 \pi}\right)^{2}
$$

where $\eta_{q}$ is the quantum efficiency, $\lambda$ is the wavelength, $P_{S}$ and $G_{S}$ are the transmit power and telescope gain of $S$, respectively, $L_{A}\left(D_{1}\right)$ is the atmospheric loss with respect to the distance $D_{1}$, and $\eta_{S}$ and $\eta_{D}$ are the optical efficiencies at $S$ and $D$, respectively.

The average received power at $D$ with respect to $\theta$ can be written as

$$
\begin{aligned}
\bar{P}_{D} & =\mathbb{E}_{\theta}\left\{K_{1} G_{D} L(\theta)\right\}=K_{1} G_{D} \mathbb{E}_{\theta}\left\{\exp \left(-G_{D} \theta^{2}\right)\right\} \\
& =K_{1} G_{D} \mathcal{M}_{\theta^{2}}\left(-G_{D}\right) .
\end{aligned}
$$

Substituting the MGF of $\theta^{2}$ in (1) into (4) yields

$$
\bar{P}_{D}=\frac{K_{1} G_{D} \exp \left(\frac{-G_{D} \mu_{V}^{2}}{1+2 \sigma_{V}^{2} G_{D}}+\frac{-G_{D} \mu_{H}^{2}}{1+2 \sigma_{H}^{2} G_{D}}\right)}{\sqrt{\left(1+2 \sigma_{V}^{2} G_{D}\right)\left(1+2 \sigma_{H}^{2} G_{D}\right)}} .
$$

\section{A. Asymptotic Result}

When $G_{D} \rightarrow \infty$, we have

$$
\begin{aligned}
& \lim _{G_{D} \rightarrow \infty} \bar{P}_{D}=\lim _{G_{D} \rightarrow \infty} \frac{K_{1} G_{D} \exp \left(\frac{-G_{D} \mu_{V}^{2}}{1+2 \sigma_{V}^{2} G_{D}}+\frac{-G_{D} \mu_{H}^{2}}{1+2 \sigma_{H}^{2} G_{D}}\right)}{\sqrt{\left(1+2 \sigma_{V}^{2} G_{D}\right)\left(1+2 \sigma_{H}^{2} G_{D}\right)}} \\
& \simeq K_{1} \frac{\exp \left(-\frac{\mu_{V}^{2}}{2 \sigma_{V}^{2}}-\frac{\mu_{H}^{2}}{2 \sigma_{H}^{2}}\right)}{\sqrt{2 \sigma_{V}^{2} 2 \sigma_{H}^{2}}}=\frac{K_{1}}{2 \sigma_{V} \sigma_{H}} \exp \left(-\frac{\mu_{V}^{2}}{2 \sigma_{V}^{2}}-\frac{\mu_{H}^{2}}{2 \sigma_{H}^{2}}\right),
\end{aligned}
$$

which shows a bound for $\bar{P}_{D}$. It implies when the telescope gain of $D$ is sufficiently large, the received power at $D$ reaches a constant asymptotic value.

\section{B. Maximization of $\bar{P}_{D}$}

When $\sigma_{V}=\sigma_{H}=\sigma$, taking the natural base of both sides in (5) yields

$$
\begin{aligned}
\ln \bar{P}_{D}= & \ln K_{1}+\ln \left(\frac{G_{D}}{1+2 \sigma^{2} G_{D}}\right) \\
& -\frac{G_{D} \mu_{V}^{2}}{1+2 \sigma^{2} G_{D}}-\frac{G_{D} \mu_{H}^{2}}{1+2 \sigma^{2} G_{D}} .
\end{aligned}
$$

Let $x=\frac{G_{D}}{1+2 \sigma^{2} G_{D}}$ and $f(x)=\ln \bar{P}_{D}$. (7) can be further written as

$$
f(x)=\ln K_{1}+\ln x-\left(\mu_{V}^{2}+\mu_{H}^{2}\right) x .
$$

Let the first derivative of $f(x)$ with respect to $x$ be equal to zero, and a unique stationary point can be obtained as

$$
\frac{\partial f(x)}{\partial x}=\frac{1}{x}-\left(\mu_{V}^{2}+\mu_{H}^{2}\right)=0 \Rightarrow x^{\star}=\frac{1}{\mu_{V}^{2}+\mu_{H}^{2}} .
$$

From the first derivative of $f(x)$, we can easily see that for $x>x^{\star}, \frac{\partial f(x)}{\partial x}<0$, and for $x<x^{\star}, \frac{\partial f(x)}{\partial x}>0$. Therefore, $f(x)$ is an increasing function over $\left(0, x^{\star}\right)$, and a decreasing function over $\left(x^{\star},+\infty\right)$. To summarize, $x^{\star}$ is the uniquely maximum point for $f(x)$. In view of the positive property of $G_{D}$ and the function property of $x=\frac{G_{D}}{1+2 \sigma^{2} G_{D}}$, the corresponding optimal point $G_{D}$ that maximizes $\bar{P}_{D}$ can be written as

$$
G_{D}^{\star}= \begin{cases}\left(\mu_{V}^{2}+\mu_{H}^{2}-2 \sigma^{2}\right)^{-1}, & \text { if } \mu_{V}^{2}+\mu_{H}^{2}>2 \sigma^{2} ; \\ +\infty, & \text { otherwise. }\end{cases}
$$

\section{Received Power at $E$}

The information intercept relies on the backflash, so the received power at $E$ is given by [10]

$$
P_{E}=P_{D}(\theta) K_{2} L\left(\theta_{E}\right) G_{D}=K_{1} K_{2} G_{D}^{2} L(\theta) L\left(\theta_{E}\right),
$$

where $L\left(\theta_{E}\right)=\exp \left(-G_{D} \theta_{E}^{2}\right), \theta_{E}=\sqrt{\left(\theta_{V}+\alpha\right)^{2}+\theta_{H}^{2}}$, and $\alpha$ is the pointing direction error angle due to backflash. In (11), $K_{2}$ is given by

$$
K_{2}=\eta_{f} \eta_{q} \eta_{B} G_{E} \eta_{E} \frac{L_{A}\left(D_{2}\right)}{D_{2}^{2}}\left(\frac{\lambda}{4 \pi}\right)^{2},
$$

where $L_{A}\left(D_{2}\right)$ is the atmospheric loss with respect to the distance $D_{2}$, and $\eta_{f}, \eta_{E}$ and $G_{E}$ are the backflash probability, optical efficiency and telescope gain of $E$, respectively.

The average received power at $E$ can be written as

$$
\begin{aligned}
& \bar{P}_{E}=\mathbb{E}\left\{K_{1} K_{2} G_{D}^{2} L(\theta) L\left(\theta_{E}\right)\right\} \\
& =K_{1} K_{2} G_{D}^{2} \mathbb{E}\left\{\exp \left(-G_{D} \theta^{2}-G_{D} \theta_{E}^{2}\right)\right\} \\
& =K_{1} K_{2} G_{D}^{2} \mathbb{E}\left\{\exp \left(-G_{D}\left(2 \theta_{V}^{2}+2 \theta_{H}^{2}+2 \alpha \theta_{V}+\alpha^{2}\right)\right)\right\},
\end{aligned}
$$

where the expectation term can be further written as

$$
\begin{aligned}
\mathbb{E} & \left\{\exp \left(-G_{D}\left(2 \theta_{V}^{2}+2 \theta_{H}^{2}+2 \alpha \theta_{V}+\alpha^{2}\right)\right)\right\} \\
= & \exp \left(-G_{D} \alpha^{2}\right) \mathbb{E}\left\{\exp \left(-2 G_{D}\left(\theta_{V}^{2}+\theta_{H}^{2}+\alpha \theta_{V}\right)\right)\right\} \\
= & \exp \left(-G_{D} \alpha^{2}\right) \mathbb{E}\left\{\exp \left(-2 G_{D} \theta_{H}^{2}\right)\right\} \\
& \mathbb{E}\left\{\exp \left(-2 G_{D}\left(\theta_{V}^{2}+\alpha \theta_{V}\right)\right)\right\} .
\end{aligned}
$$


By using the probability density functions of $\theta_{V}$ and $\theta_{H}$, closed-form expressions for the two expectation operators in (14) can be derived as

$$
\begin{aligned}
& \mathbb{E}_{\theta_{E}}\left\{\exp \left(-2 G_{D}\left(\theta_{V}^{2}+\alpha \theta_{V}\right)\right)\right\} \\
& =\frac{\exp \left(-\frac{\mu_{V}^{2}}{2 \sigma_{V}^{2}}\right)}{\sqrt{4 G_{D} \sigma_{V}^{2}+1}} \exp \left(\frac{\left(2 \alpha G_{D}-\frac{\mu_{V}}{\sigma_{V}^{2}}\right)^{2}}{8 G_{D}+\frac{2}{\sigma_{V}^{2}}}\right), \\
& \mathbb{E}_{\theta_{E}}\left\{\exp \left(-2 G_{D} \theta_{H}^{2}\right)\right\} \\
& =\frac{\exp \left(-\frac{\mu_{H}^{2}}{2 \sigma_{H}^{2}}\right)}{\sqrt{4 \sigma_{H}^{2} G_{D}+1}} \exp \left(\frac{\left(\frac{\mu_{H}}{\sigma_{H}^{2}}\right)^{2}}{8 G_{D}+\frac{2}{\sigma_{H}^{2}}}\right)
\end{aligned}
$$

respectively.

A closed-form expression for $\bar{P}_{E}$ can finally be derived for arbitrary $\mu_{V}, \mu_{H}, \sigma_{V}, \sigma_{H}$, and $\alpha$ as

$$
\begin{aligned}
\bar{P}_{E}= & \frac{K_{1} K_{2} G_{D}^{2} \exp \left(-\frac{\mu_{V}^{2}}{2 \sigma_{V}^{2}}-\frac{\mu_{H}^{2}}{2 \sigma_{H}^{2}}\right)}{\sqrt{\left(4 G_{D} \sigma_{V}^{2}+1\right)\left(4 \sigma_{H}^{2} G_{D}+1\right)}} \\
& \exp \left(\frac{\left(2 \alpha G_{D}-\frac{\mu_{V}}{\sigma_{V}^{2}}\right)^{2}}{8 G_{D}+\frac{2}{\sigma_{V}^{2}}}+\frac{\left(\frac{\mu_{H}}{\sigma_{H}^{2}}\right)^{2}}{8 G_{D}+\frac{2}{\sigma_{H}^{2}}}-G_{D} \alpha^{2}\right) .
\end{aligned}
$$

\section{A. Special Cases}

If $\alpha=0$, which means that $E$ is in the vicinity of $S, \bar{P}_{E}$ becomes

$$
\begin{aligned}
\bar{P}_{E} & =K_{1} K_{2} G_{D}^{2} \mathbb{E}\left\{\exp \left(-2 G_{D}\left(\theta_{V}^{2}+\theta_{H}^{2}\right)\right)\right\} \\
& =\frac{K_{1} K_{2} G_{D}^{2} \exp \left(\frac{-2 \mu_{V}^{2} G_{D}}{1+4 \sigma_{V}^{2} G_{D}}-\frac{2 \mu_{H}^{2} G_{D}}{1+4 \sigma_{H}^{2} G_{D}}\right)}{\sqrt{\left(1+4 \sigma_{V}^{2} G_{D}\right)\left(1+4 \sigma_{H}^{2} G_{D}\right)}} .
\end{aligned}
$$

In the $\alpha=0$ case, when $G_{D} \rightarrow+\infty, \bar{P}_{E}$ is approximately equal to

$$
\begin{aligned}
& \lim _{G_{D} \rightarrow+\infty} \frac{K_{1} K_{2} G_{D}^{2} \exp \left(\frac{-2 \mu_{V}^{2} G_{D}}{1+4 \sigma_{V}^{2} G_{D}}-\frac{2 \mu_{H}^{2} G_{D}}{1+4 \sigma_{H}^{2} G_{D}}\right)}{\sqrt{\left(1+4 \sigma_{V}^{2} G_{D}\right)\left(1+4 \sigma_{H}^{2} G_{D}\right)}} \\
& \simeq \frac{K_{1} K_{2} \exp \left(-\frac{\mu_{V}^{2}}{2 \sigma_{V}^{2}}-\frac{\mu_{H}^{2}}{2 \sigma_{H}^{2}}\right)}{4 \sigma_{V} \sigma_{H}} G_{D},
\end{aligned}
$$

which shows that $\bar{P}_{E}$ is approximately proportional to $G_{D}$ for $G_{D} \rightarrow+\infty$ and $\alpha=0$.

\section{B. Maximization of $\bar{P}_{E}$}

For $\mu_{V}=\mu_{H}=0$ and $\sigma_{V}=\sigma_{H}=\sigma, \bar{P}_{E}$ can be simplified as

$$
\bar{P}_{E}=\frac{K_{1} K_{2} G_{D}^{2}}{4 \sigma^{2} G_{D}+1} \exp \left(\frac{2 \sigma^{2} \alpha^{2} G_{D}^{2}}{4 \sigma^{2} G_{D}+1}-G_{D} \alpha^{2}\right) .
$$

The first derivative of $\bar{P}_{E}$ with respect to $G_{D}$ is given by (21), where the positive or negative value depends only on the last term, i.e.,

$$
\begin{aligned}
\Theta= & -16 \sigma^{4} \alpha^{2} G_{D}^{3}+\left(32 \sigma^{4}-8 \sigma^{2} \alpha^{2}\right) G_{D}^{2} \\
& +\left(24 \sigma^{2}-2 \alpha^{2}\right) G_{D}+4,
\end{aligned}
$$

which is a standard univariate cubic equation with respect to $G_{D}$. If $\sigma$ and $\alpha$ are known, we can easily find the roots for $\Theta=0$ and the positive and negative value intervals for $\frac{\partial \bar{P}_{E}}{\partial G_{D}}$, and thereby finding the extreme points for $\bar{P}_{E}$. Specially, when $G_{D}=0, \Theta=4$ is positive, so over $G_{D} \in(0,+\infty), \bar{P}_{E}$ must increase first. The trend and extreme point of $\bar{P}_{E}$ are very useful and important for the secure system design.

\section{Ratio of $\bar{P}_{E}$ to $\bar{P}_{D}$}

An important metric for the secure analysis is the ratio of $\bar{P}_{E}$ to $\bar{P}_{D}$, which can be easily derived by using the closedform expressions for $\bar{P}_{E}$ and $\bar{P}_{D}$, is given by

$$
\begin{aligned}
\Delta= & \frac{\bar{P}_{E}}{\bar{P}_{D}}=\frac{K_{2} G_{D} \sqrt{\left(1+2 \sigma_{V}^{2} G_{D}\right)\left(1+2 \sigma_{H}^{2} G_{D}\right)}}{\sqrt{\left(4 \sigma_{V}^{2} G_{D}+1\right)\left(4 \sigma_{H}^{2} G_{D}+1\right)}} \\
& \exp \left(-\frac{\mu_{V}^{2}}{2 \sigma_{V}^{2}}-\frac{\mu_{H}^{2}}{2 \sigma_{H}^{2}}\right) \exp \left(\frac{G_{D} \mu_{V}^{2}}{1+2 \sigma_{V}^{2} G_{D}}+\frac{G_{D} \mu_{H}^{2}}{1+2 \sigma_{H}^{2} G_{D}}\right) \\
& \exp \left(\frac{\left(2 \alpha G_{D}-\frac{\mu_{V}}{\sigma_{V}^{2}}\right)^{2}}{8 G_{D}+\frac{2}{\sigma_{V}^{2}}}+\frac{\left(\frac{\mu_{H}}{\sigma_{H}^{2}}\right)^{2}}{8 G_{D}+\frac{2}{\sigma_{H}^{2}}}-\alpha^{2} G_{D}\right) .
\end{aligned}
$$

This expression is relatively complicated, and does not provide us some useful insights for the secure system design. Here, we consider some special cases to analyze this ratio.

For $\mu_{V}=\mu_{H}=0$ and $\sigma_{V}=\sigma_{H}=\sigma$, the expression for $\Delta$ can be simplified as

$$
\Delta=\frac{\left(1+2 \sigma^{2} G_{D}\right) K_{2} G_{D}}{4 \sigma^{2} G_{D}+1} \exp \left(\frac{\alpha^{2} G_{D}^{2}}{2 G_{D}+\frac{1}{2 \sigma^{2}}}-\alpha^{2} G_{D}\right) .
$$

The first derivative of $\Delta$ with respect to $G_{D}$ is shown in (25), where the positive or negative value of $\frac{\partial \Delta}{\partial G_{D}}$ depends only on $\Theta_{2}$, a standard univariate quartic equation. If $\alpha$ and $\sigma$ are known, the roots for $\Theta_{2}=0$ can be easily derived, and the positive and negative value intervals can be also determined. Thus, we can analyze the changing trend of $\Delta$ and derive the extreme points.

When $\alpha=0, \mu_{V}=\mu_{H}=0$ and $\sigma_{V}=\sigma_{H}=\sigma, \Delta$ becomes

$$
\Delta=\frac{\left(1+2 \sigma^{2} G_{D}\right) K_{2} G_{D}}{4 \sigma^{2} G_{D}+1} .
$$

The first derivative of $\Delta$ with respect to $G_{D}$ in (26) is

$$
\frac{\partial \Delta}{\partial G_{D}}=\frac{K_{2}+4 \sigma^{2} K_{2} G_{D}^{2}+8 \sigma^{4} K_{2} G_{D}^{2}\left(2 G_{D}-1\right)}{\left(4 \sigma^{2} G_{D}+1\right)^{2}} .
$$

It is obvious that for $G_{D}>\frac{1}{2}, \frac{\partial \Delta}{\partial G_{D}}$ must be positive. As $G_{D} \gg 1$ in the most practical cases, we can treat $\frac{\partial \Delta}{\partial G_{D}}$ as a positive value, and therefore, $\Delta$ always increases as $G_{D}$ grows. If $G_{D}$ is sufficiently large, $\Delta$ can be approximately obtained by

$$
\Delta \simeq \frac{K_{2} G_{D}}{2}
$$




$$
\frac{\partial \bar{P}_{E}}{\partial G_{D}}=\frac{K_{1} K_{2} G_{D} \exp \left(-\frac{\alpha^{2} G_{D}\left(4 \sigma^{2} G_{D}+2\right)}{8 \sigma^{2} G_{D}+2}\right)}{2\left(4 \sigma^{2} G_{D}+1\right)^{3}}\left[-\left(4 \sigma^{2}\right)^{2} \alpha^{2} G_{D}^{3}+\left(2\left(4 \sigma^{2}\right)^{2}-2 \cdot 4 \sigma^{2} \alpha^{2}\right) G_{D}^{2}+\left(6 \cdot 4 \sigma^{2}-2 \alpha^{2}\right) G_{D}+4\right]
$$

$\frac{\partial \Delta}{\partial G_{D}}=\frac{K_{2} \exp \left(-\frac{\alpha^{2} G_{D}\left(2 \sigma^{2} G_{D}+1\right)}{4 \sigma^{2} G_{D}+1}\right)}{\left(4 \sigma^{2} G_{D}+1\right)^{3}} \underbrace{\left[-16 \alpha^{2} \sigma^{6} G_{D}^{4}-\left(16 \sigma^{4} \alpha^{2}-32 \sigma^{6}\right) G_{D}^{3}-\left(6 \sigma^{2} \alpha^{2}-24 \sigma^{4}\right) G_{D}^{2}-\left(\alpha^{2}-8 \sigma^{2}\right) G_{D}+1\right]}_{\Theta_{2}}$.

\section{NUMERICAL RESUltS}

To simplify the parameter setting, $D_{1}=D_{2}=900 \mathrm{~km}$, $L_{A}(D 1)=L_{A}(D 2)=0.5, \lambda=780 \mathrm{~nm}, P_{S}=0 \mathrm{~dB}, G_{S}=$ $G_{E}=10^{11}, \eta_{f}=0.04, \eta_{q}=0.1$, and $\eta_{S}=\eta_{D}=0.9$ are assumed in the following simulation results. In each MonteCarlo simulation result, $10^{7}$ realizations are generated to get the corresponding average value according to the statistical properties, i.e., $\mu_{V}, \mu_{H}, \sigma_{V}$ and $\sigma_{H}$.

As shown in Fig. 1, the received power at $D$ increases as $G_{D}$ grows, due to the improved telescope gain. When $G_{D}$ is sufficiently large, $\bar{P}_{D}$ reaches an asymptotic bound, as proved in (6). It is obvious that $\bar{P}_{D}$ is improved with increasing $\mu_{H}$, because a smaller $\mu_{H}$ means a better pointing direction. The maximum points are also marked in Fig. 1, which are derived from the maximization analysis for $\bar{P}_{D}$, where the maximum points for $\mu_{V}^{2}+\mu_{H}^{2}<2 \sigma^{2}$ are infinity.

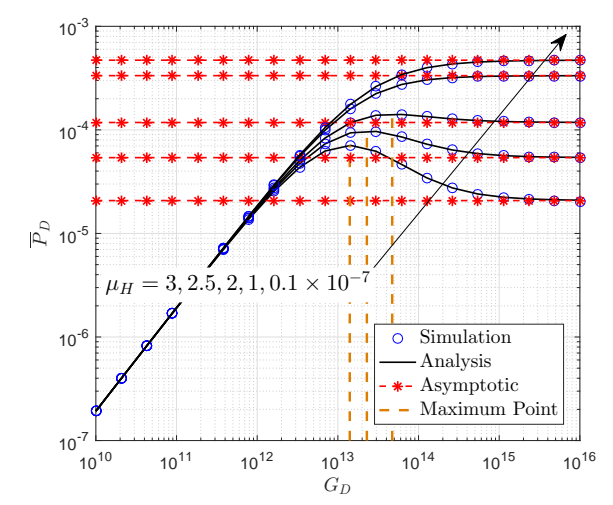

Fig. 1. $\bar{P}_{D}$ versus $G_{D}$ with various values of $\mu_{H}$ for $\sigma_{V}^{2}=\sigma_{H}^{2}=$ $1.44 \times 10^{-14}$, and $\mu_{V}=1 \times 10^{-7}$.

Fig. 2 plots $\bar{P}_{E}$ versus $G_{D}$ for different values of $\alpha . \bar{P}_{E}$ for $\alpha>0$ increases first, and reaches a vertex before a sharp decline, while $\bar{P}_{E}$ for $\alpha=0$ always increases with increasing $G_{D}$, where the asymptotic results are derived by (19). When $G_{D}$ is sufficiently large, $\bar{P}_{E}$ becomes lower as $\alpha$ grows, because a larger $\alpha$ means a larger distance between the transmitter and eavesdropper. The maximum points in Fig. 2 are obtained in the analysis of Subsection IV-B.

To validate the correctness of (17) for the general pointing errors, we also vary the values of $\mu_{V}$ and $\sigma_{V}^{2}$ in Figs. 3-4, respectively. From Figs. 3-4, we can easily see that a large

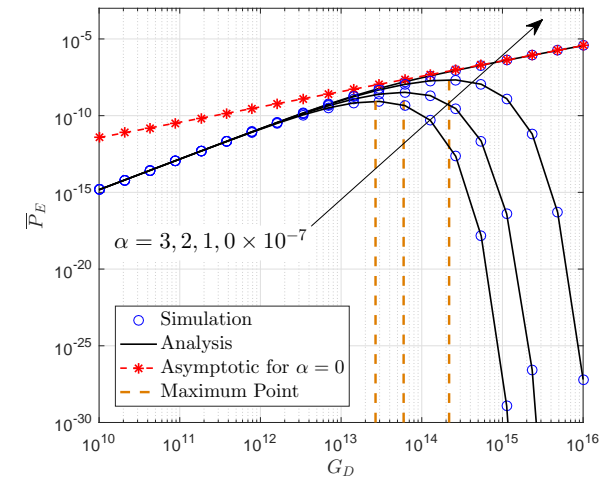

Fig. 2. $\bar{P}_{E}$ versus $G_{D}$ with various values of $\alpha$ for $\sigma_{V}^{2}=\sigma_{H}^{2}=1 \times 10^{-14}$, and $\mu_{V}=\mu_{H}=0$.

$\mu_{V}$ (or $\sigma_{V}^{2}$ ) results in a larger $\bar{P}_{E}$. More specifically, $\bar{P}_{E}$ under different values of $\mu_{V}$ almost converges in the low and high $G_{D}$ regions, and the gap is only obvious in the medium $G_{D}$ region. In the $\sigma_{V}^{2}$ changing case, the gap of $\bar{P}_{E}$ under different $\sigma_{V}^{2}$ becomes larger with increasing $G_{D}$.

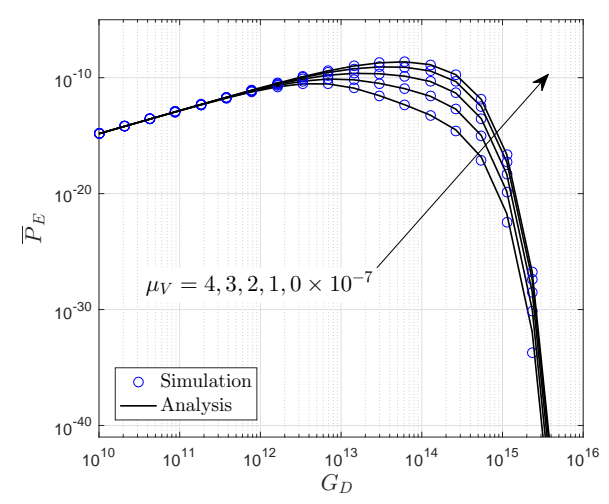

Fig. 3. $\bar{P}_{E}$ versus $G_{D}$ with various values of $\mu_{V}$ for $\sigma_{V}^{2}=\sigma_{H}^{2}=$ $1 \times 10^{-14}, \mu_{H}=1 \times 10^{-7}$, and $\alpha=2 \times 10^{-7}$.

In Fig. 5, $\Delta$ for $\alpha>0$ increases first before achieving a vertex after which this figure is rapidly falling, rather than a continuous growth in the figure for $\alpha=0$, where the asymptotic results are obtained by (28). The maximum points for (24) are also presented in Fig. 5, where the decreasing trend of maximum points are obvious with increasing $\alpha$.

To present more general pointing error cases, we consider 


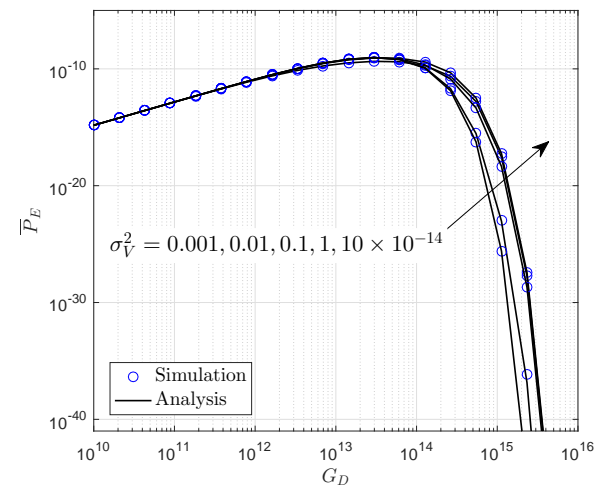

Fig. 4. $\bar{P}_{E}$ versus $G_{D}$ with various values of $\sigma_{V}^{2}$ for $\sigma_{H}^{2}=1 \times 10^{-14}$, $\mu_{V}=\mu_{H}=1 \times 10^{-7}$, and $\alpha=2 \times 10^{-7}$.

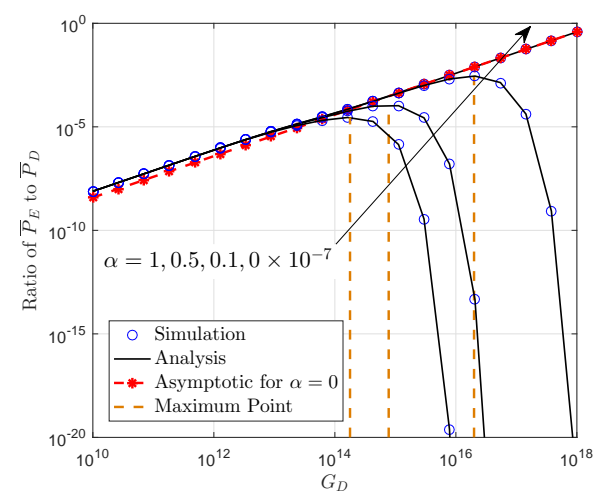

Fig. 5. $\Delta$ versus $G_{D}$ with various values of $\alpha$ for $\sigma_{V}^{2}=\sigma_{H}^{2}=1 \times 10^{-14}$, and $\mu_{V}=\mu_{H}=0$.

different values of $\mu_{V}$ and $\mu_{H}$, and set the same variance of $\theta_{V}$ and $\theta_{H}$ in Fig. 6. There is an increasing trend of $\Delta$ as the variance grows, although the difference of $\Delta$ among different variances is not obvious in the low $G_{D}$ region.

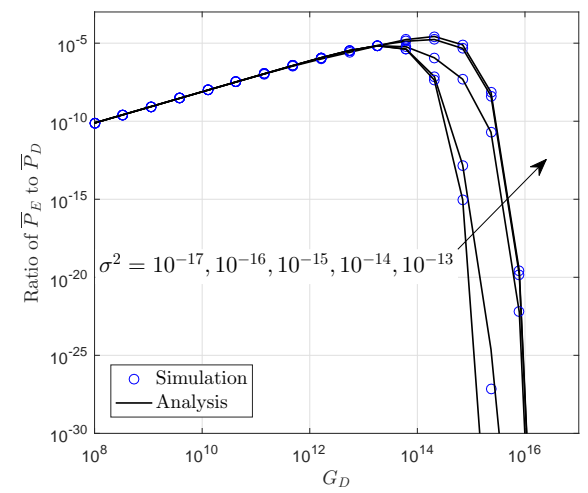

Fig. 6. $\Delta$ versus $G_{D}$ with various values of $\sigma_{V}^{2}=\sigma_{H}^{2}=\sigma^{2}$ for $\mu_{V}=$ $1 \times 10^{-7}, \mu_{H}=0$, and $\alpha=1 \times 10^{-7}$.

\section{CONCLUSION}

In this letter, closed-form expressions for $\bar{P}_{D}, \bar{P}_{E}$, and $\Delta$ were derived, and simplified expressions in some special cases were also given. To get more design insights, we performed an analytical maximization for $\bar{P}_{D}, \bar{P}_{E}$, and $\Delta$ based on some special settings of some selected parameters. In the numerical section, we used the Monte-Carlo simulations to validate the correctness of our newly derived expressions. From both the derived expressions and numerical results, we can get some design guidelines for the telescope gain at the legitimate receiver to achieve a specific purpose, shown as follows:

- If the legitimate receiver only wants to improve its received power (without taking the eavesdropper into account) in the $\sigma_{V}^{2}=\sigma_{H}^{2}$ case, the optimal $G_{D}$ can be found by (10), which depends on the parameter setting, i.e., may not just make $G_{D}$ as large as possible (shown in Fig. 1).

- If we only want to make $\bar{P}_{E}$ lower, the legitimate receiver should avoid the optimal $G_{D}$ for $\bar{P}_{E}$, where the impact of $\bar{P}_{D}$ is neglected due to the priority of security. From Fig. 2, we should make $G_{D}$ as small (or large) as possible to avoid the vertex for $\alpha \neq 0$. When $\alpha=0$, decreasing $G_{D}$ is the only way to get better security.

- If we need to balance the received power performance between the legitimate receiver and eavesdropper, $\Delta$ should be taken into account, which reflects the joint effect of $G_{D}$ on $\bar{P}_{D}$ and $\bar{P}_{E}$. From Figs. 5-6, to make $\bar{P}_{D}$ larger and $\bar{P}_{E}$ smaller for $\alpha \neq 0$, the legitimate receiver can increase $G_{D}$ until arriving to a specific threshold. If $\alpha=0$, we have no choice but to decrease $G_{D}$.

\section{REFERENCES}

[1] C. E. Shannon, "Communication theory of secrecy systems," Bell Syst. Tech. J., vol. 28, no. 4, pp. 656-715, 1949.

[2] P. V. Trinh, A. T. Pham, A. Carrasco-Casado, and M. Toyoshima, "Quantum key distribution over FSO: Current development and future perspectives," in Proc. Electromagnetics Research Symposium (PIERSToyama), Aug. 2018, pp. 1672-1679.

[3] C. Cheng, R. Lu, A. Petzoldt, and T. Takagi, "Securing the internet of things in a quantum world," IEEE Commun. Mag., vol. 55, no. 2, pp. 116-120, 2017.

[4] K. Inoue, "Quantum key distribution technologies," IEEE J. Sel. Topics Quantum Electron., vol. 12, no. 4, pp. 888-896, Aug. 2006.

[5] S. Arnon, "Effects of atmospheric turbulence and building sway on optical wireless-communication systems," Opt. Lett., vol. 28, no. 2, pp. 129-131, Jan. 2003.

[6] I. S. Ansari, M.-S. Alouini, and J. Cheng, "Ergodic capacity analysis of free-space optical links with nonzeros boresight pointing errors," IEEE Trans. Wireless Commun., vol. 14, no. 8, pp. 4248-4264, Aug. 2015.

[7] H. AlQuwaiee, H.-C. Yang, and M.-S. Alouini, "On the asymptotic capacity of dual-aperture FSO systems with generalzied pointing error model," IEEE Trans. Wireless Commun., vol. 15, no. 9, pp. 6502-6512, Sep. 2016.

[8] A. Lacaita, S. Cova, A. Spinelli, and F. Zappa, "Photon-assisted avalanche spreading in reach-through photodiodes," Appl. Phys. Lett., vol. 62, no. 6, pp. 606-608, Feb. 1993.

[9] A. Meda, I. P. Degiovanni, A. Tosi, Z. Yuan, G. Brida, and M. Genovese, "Quantifying backflash radiation to prevent zero-error attacks in quantum key distribution," Light Sci. Appl., vol. 6, no. pp. e16261, 2017.

[10] J. Kupferman, and S. Arnon, "Zero-error attacks on a quantum key distribution FSO system," OSA Continuum, vol. 1, no. 3, pp. 1079-1086, Nov. 2018.

[11] M. K. Simon and M.-S. Alouini, Digital Communication over Fading Channels. New York, NY, USA: Wiley, 2000.

[12] P. Pena-Martin, J. M. Romero-Jerez, and F. J. Lopez-Martinez, "Generalized MGF of Beckmann fading with applications to wireless communications performance analysis," IEEE Trans. Commun., vol. 65, no. 9 , pp. 3933-3943, Sep. 2017. 